\title{
Study on the Structure and Behavioral Choice of IDs Model
}

\author{
Zhangyani \\ Department of Computer Science,Qiannan Normal College for Nationalities,Duyun,Guizhou, china, 558000 \\ ad.wang@qq.com
}

\begin{abstract}
The complicated decision making problem is one of the important components for the study on the system of artificial intelligence area. This thesis, based on the Bayesian technology and decision-making theory, is going to optimize the traditional IDs model and improve the ability of expression of the model. Firstly, the structure decomposition method is given to divide the IDs into two parts, which are probability network structure and utility function structure. Secondly, a new MDL evaluation standard is put forward to reduce the dependence on statistics of the traditional MDL evaluation standard and based on the new standard to propose to use the PS-EM in the model choice of probability network structure; and also by using the sum of individual utility function instead of the joint utility function to create the BP neural network to study the utility function structure of the IDs. The experimental result shows the method mentioned above is effective.
\end{abstract} choice

Keywords-Complex System, IDs, structural choice, Behavior

\section{INTRODUCTION}

Even though Bayes network has obvious advantage on modeling and inference of uncertainty problem, lacking of MDL evaluation standard makes it not so ideal on knowledge expression, decision making and behavioral interaction of multi-agent system.

IDs integrated the formalization of Agent beliefs and behavior evaluation mechanism and has become one of the most important knowledge representation tool of decision making problem ${ }^{[1][2]}$, and also applied in the air defense system construction ${ }^{[3]}$, military decision analysis, and unmanned air combat simulation ${ }^{[4]}$ and aviation image automatic understand ${ }^{[5]}$.

In the treatment of the static decision making problems, IDs is more intuitive than the decision tree, it can reveal the dependency relationship of internal structure in system, and more important thing is that IDs is much stronger than the decision tree to solve large-scale decision problems. As the growth of the number of variables, the scale of decision tree is under exponential growth, but the scale of IDs is under linear growth. In addition, The IDs can model the relationship among discrete, continuous and compound variables, which is incapable for decision tree. ${ }^{[6][7]}$

The reasoning of IDs is mostly based on the joint tree propagation method, but the triangularization operation in the construction of joint tree is still an open problem for research; moreover, the joint tree cannot avoid completely the high-dimensional problems occurred in the process of reasoning in the calculation, thus reasoning of complex system is still a NP problem. ${ }^{[8][9]}$

This thesis is aiming at solving the problem among data dependence, computational complexity and the problem of non-probability relationship through the IDs model choice by putting forward PS - EM algorithm to processing the model selection of the probability structure of IDs; setup a BP neural network by which can learn the partial utility function to achieve the model selection on structure division. PS - EM algorithm by introducing a kind of fusion prior knowledge of MDL score (PS - MDL) to reduce the traditional MDL score on the dependence of the data, improve the computational efficiency by separately the parameters and structure of the score calculation.

\section{IDS MODEL}

Due to the IDs model is a decision model combined of probability graph model and utility mechanism, first we introduce the utility theory as follow:

\section{A. Descriptyion for IDs Model}

IDs is a directed acyclic graph and can be defined as a triad $I D=(G, P, U)$, among it $G=(V, E)$ is directed acyclic graph constituted by the random node, decision node and utility node, and the edge set $\mathrm{E}$ for the connection of all the nodes.

IF $X=\left\{X_{1}, \ldots, X_{n}\right\}$ is stochastic variable sets and $D=\left\{D_{1}, \ldots, D_{k}\right\}$ is decision variable set, $\mathrm{U}$ is the partial utility function set $U=\left\{U_{1}, \ldots, U_{k}\right\}$, and suppose every decision variable is corresponding a stochastic variable, that is the partial utility function for $D_{i}$ is $U_{i}$ 。

The IDs is formulated by Structure strategy sub model, Probability sub model and Utility sub model, following are the description of the above sub models:

(1)Structure Strategy model Structure Strategy model confirm $\delta_{i}$ for each $D_{i}$, and $\delta_{i}$ is a function between $\operatorname{Pa}\left(D_{i}\right)$ and a selectable behavior. The strategy for IDs is $\delta=\left\{\delta_{i}\right\}, i \in[1: k]$ can be defined as $\Omega_{P a(D)} \rightarrow \Omega_{D}$, $\Omega_{P a(D)}$ indicates the REF for $\operatorname{Pa}\left(D_{i}\right), \Omega_{D}$ indicates the action space of $D_{i}$ 
(2)Probability model when the behavior of $D_{i}$ is given, joint probability distribution of random variables is bayesian network joint probability distribution. Given each variable $X_{i}$ a father node set $\operatorname{Pa}\left(X_{i}\right)$, the local condition probability distribution for $X_{i}$ is $P\left(X_{i} \mid \operatorname{Pa}\left(X_{i}\right)\right)$. Take Decision node as random node, for a given decision rule $\delta_{i}$, $D_{i}$ has the following condition probability distribution:

$$
P_{\delta_{i}}\left(D_{i} \mid P a\left(D_{i}\right)\right)= \begin{cases}1 & \text { 当 } \delta_{i}\left(P a\left(D_{i}\right)\right)=D_{i} \\ 0 & \text { 否则 }\end{cases}
$$

Random variable and decision variable of the joint probability distribution can be expressed as:

$$
P(X, D)=\prod_{i=1}^{n} P\left(X_{i} \mid \operatorname{Pa}\left(X_{i}\right)\right) \prod_{j=1}^{k} P_{\delta_{i}}\left(D_{i} \mid \operatorname{Pa}\left(D_{i}\right)\right)
$$

(3) The utility model Utility function is a function that contact decision node and the state variable. For each $U_{i}$ there is a $U_{i}\left(\operatorname{Pa}\left(U_{i}\right)\right)$ which is related with $\operatorname{Pa}\left(U_{i}\right)$. The Sum of all local utility can be represented as:

$$
U(X, D)=\sum_{i=1}^{k} U_{i}\left(\operatorname{Pa}\left(U_{i}\right)\right)
$$

$U_{\delta}$ can be represented as:

$$
U_{\delta}=\sum_{X, D} P(X, D) \sum_{i=1}^{k} U_{i}\left(P a\left(U_{i}\right)\right.
$$

If the system random node is implied, the $\mathrm{X}$ represents the set of state variables and observation variable.

Following is an example to show the usage of IDs:

If an oil profiteer wants to drill oil (Drill) on some land, the uncertain factors for him as: Drilling expenses and the inventory of the oil. Because we only can get the information of oil inventory (Result) by testing first (Test) in order to make decision on whether to dill or not, then whether to do the test or not become the most important decision making problem. The Oil Wildcatter as below:

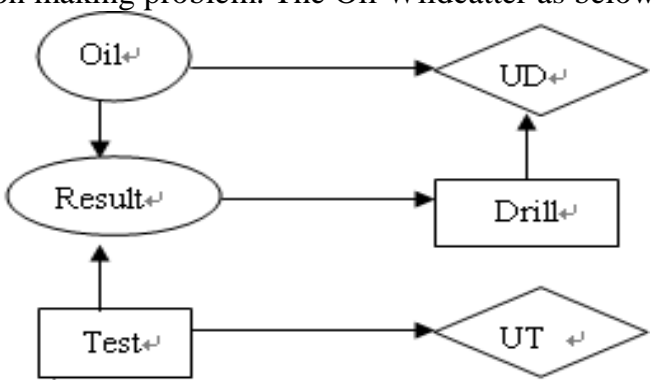

Chart 1 Oil Wildcatter Model

(1) Indication for each of the node from above chart 1:

(1)random node

Oil: Oil indicates the oil content of the land, the possible REF as dry, wet、soak;
Result: indicates the testing result, REF as no structure(Ns)、 open structure(Os)、 closed structure(Cs)、 no result,

(2)Decision Node

Drill: REF for drill as not drill and drill

Test: REF for test as not test and test

(3)Utility node:

UD: UD is the utility function for drill and related with oil and drill;

UT: UT is the utility function for test and related with test;

(2) utility node to utility function:

Table 1 Utility function for node drill

\begin{tabular}{|c|c|c|c|}
\hline & dry & wet & soak \\
\hline Not drill & $\$ 0$ & $\$ 0$ & $\$ 0$ \\
\hline Drill & $-\$ 70$ & $\$ 50$ & $\$ 200$ \\
\hline
\end{tabular}

Table 2 Utility function for node Test

\begin{tabular}{|c|c|c|c|}
\hline & Ns & Os & Cs \\
\hline Not test & $\$ 0$ & $\$ 0$ & $\$ 0$ \\
\hline Test & $-\$ 10$ & $-\$ 10$ & $-\$ 10$ \\
\hline
\end{tabular}

According to chart1, if not drill, whatever the ref for oil is, the utility value for profiteer is 0 . If drill, when the REF for oil are dry 、 wet s soak, the utility value for profiteer are -70、50、200. Similar with drill node, chart 2 shows the utility function for Test node.

\section{B. Decomposition of IDs Structure}

If $V_{B}$ is the node set from IDs node set $\mathrm{V}$ after taking away all the utility node, then $V_{B}$ can be considered as probability network node set under $\delta=\left(\delta_{1}, \ldots, \delta_{k}\right)$.

Between Utility node and random node is not probability dependency relationship, but a function relation, so you can't use the current method that study probability structure relation to study the structure of the utility part; In addition, the utility node easily separated from the IDs structure, this is because the utility node has no child node, the decomposition won't destroy the internal structure of the network, and even can make the structure of network becomes simple and easy to realize model selection.Chart 2 Bayesian network after taking away the utility node in Chart 1

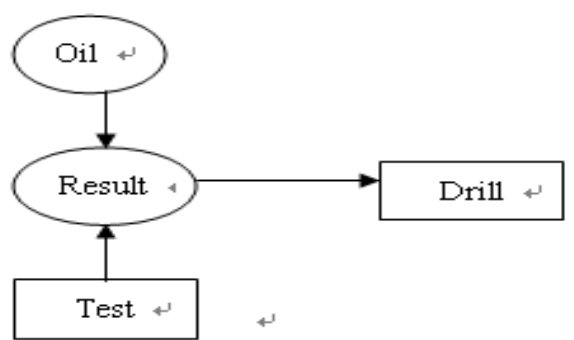

Chater 2 Bayesian network of Oil Wildcatter Model 


\section{IDS MODEL STRUCTURE CHOICE}

The commonly used probability network structure score standard has two kinds: BDe score and MDL score. BDe rating depends on the prior distribution of the model parameters, the dependence of the data is relatively lower, but lean to choose complex network structure, with high computational complexity; MDL score bias in the selection of simple network structure, the computational complexity is low, but with no prior knowledge of using network structure, the dependence on the data is much more that requires a big scope and integrity of data without much deviation $^{[12]}$. This paper combined the thoughts of with BDe score and MDL score to put forward a kind of fusion structure prior knowledge of MDL score for structure score.

\section{A. Evaluation Function for IDs Structure}

PS - EM algorithm by introducing a kind of fusion prior knowledge of MDL score (PS - MDL) to reduce the traditional MDL score on the dependence of the data, improve the computational efficiency by separately the parameters and structure of the score calculation. The total length of Fusion structure prior knowledge network structure description has four parts: length of sample description, length of existing generation network structure description, length of existing network structure description and complicated structure of the penalty term. Traditional MDL score contains only the first two parts.

If $X=\left\{X_{1}, \ldots, X_{n}\right\}$ is the random variable set of the network, $S=\left\{x^{1}, \ldots, x^{N}\right\}$ is the sample set for $X$, the set value $\left\{x_{i}^{1}, x_{i}^{2}, \ldots, x_{i}^{n_{i}}\right\}$ for each $X_{i} \in X$, the description length for each part as:

(1) length of sample description

IF sample set $S$ was complete, $N_{X}(X=x)$ refers to the amount of sample $X=x$ in $S$,the description as :

$$
H\left(X_{i} \mid \operatorname{Pa}\left(X_{i}\right)\right)=-\sum_{X_{i} \in X} \sum_{X_{i}, P a\left(X_{i}\right)} N\left(X_{i}, \operatorname{Pa}\left(X_{i}\right)\right) \log \left(\theta_{X_{i} \mid \operatorname{Pa}\left(X_{i}\right)}\right)
$$

Among that $H\left(X_{i} \mid P d\left(X_{i}\right)\right)$ is information entropy;

$$
, \theta_{X_{i} \mid \operatorname{Pa}\left(X_{i}\right)}=\frac{E\left[N\left(X_{i}, P a\left(X_{i}\right) \mid X_{i}=x_{i}^{j}\right)\right]}{E\left[N\left(P a\left(X_{i}\right) \mid X_{i}=X_{i}^{j}\right]\right.} 。
$$

( 2 ) length of existing generation network structure description

If the number of variable is $n$, constant is $d$, the formula is:

$$
\sum_{x_{i} \in X}\left[k_{i} \log _{2}(n)+d\left(n_{i}-1\right) \prod_{X_{j} \in \operatorname{Pa}\left(X_{i}\right)} n_{j}\right]
$$

That $n_{i}$ is the condition number of $X_{i}, k_{i}$ is the number

of father node for $X_{i}$ ( 3 ) length of description on difference of existing network structure description existing generation network structure
To suppose there is description for a current generation network structure $\left(B_{N}\right)$, the existing network structure $\left(B_{E}\right)$ only describes the difference aspect with $B_{N}: 1$ ) Reverse edges, (both $B_{E}$ and $B_{N}$ have but different direction.); 2) the lost edge from $B_{E}$ ( $B_{N}$ has, but $B_{E}$ does not have.); 3) the added edge for $B_{E}$ ( $B_{E}$ has, but $B_{N}$ does not have). For each node in $B_{E}$, when $B_{N}$ is given, if $r_{i}, a_{i}, m_{i}$ are the number of the reverse, lost and added edge of $B_{N}$, Length of description on difference of existing network structure and existing generation network structure can be represented as:

$$
\sum_{X_{i} \in X}\left[\left(r_{i}+m_{i}+a_{i}\right) \log _{2}[n(n-1)]\right]
$$

The result of $r_{i} 、 a_{i}$ and $m_{i}$ can be got by test,. There are $n$ nodes, the number of possible edge will be $n(n-1)$.

Length of description on difference of existing network structure description existing generation network structure

can be thought of as kind of punishment for using loss rate sample learning network structure and cause significant change of existing network structure, tend to select a best network structure that has less difference, actually it is kind of protection to the best network structure.

(4) complicated structure of the penalty term

$$
\sum_{X_{i} \in X} \frac{\log N}{2} B\left(X_{i}, \operatorname{Pa}\left(X_{i}\right) \mid X_{i}=X_{i}^{j}\right)
$$

$B\left(X_{i}, P a\left(X_{i} \mid X_{i}\right)\right)$ Refers the number of parameter required by $P\left(X_{i} \mid P d\left(X_{i}\right)\right)$. Complicated structure of the penalty term makes the PS-MDL score to find a simple network that can balance the accuracy and complexity of the network, avoiding the overdo matching.

So the Score, the length of description for B is:

$$
\begin{gathered}
\operatorname{Scor}(B: S)=\sum_{X_{i} \in X} \operatorname{Scor}_{X_{i}}\left(\theta_{i}, \operatorname{Pa}\left(X_{i}\right): S\right) \\
H\left(X_{i} \mid \operatorname{Pa}\left(X_{i}\right)\right)+\sum_{X_{i} \in X}\left[\left(r_{i}+m_{i}+a_{i}\right) \log _{2}[n(n-1)]\right]+ \\
\sum_{x_{i} \in X}\left[k_{i} \log _{2}(n)+\left(n_{i}-1\right) \prod_{X_{j} \in \operatorname{Pa}\left(X_{i}\right)} n_{j}\right] \\
\sum_{X_{i} \in X} \frac{\log N}{2} B\left(X_{i}, \operatorname{Pa}\left(X_{i}\right) \mid X_{i}=x_{i}^{j}\right)
\end{gathered}
$$

Each proof can be thought of as new data from environment 。 However the existing network structure is from the domain expert or before learning, and they are independent from each other, so their trace length can be calculated respectively. This study is to optimize existing network structure, enabling to reduce the dependence of the 
data.

\section{B. Selection algorithm of IDs model}

Compare to traditional EM algorithm, PS-EM algorithm has its advantage. PS-EM algorithm can be used in the study of parameter and structure. The basic process as: firstly to maximize the parameter with EM algorithm, then use PSMD score to make the model selection. Among them, the given initial model is $M^{0}, M^{i}(i>0)$ is generated in the NO. $i$ Time of EM algorithm, and the sequence number of the model is same as and iterative times

algorithm can be descript as below:

Given initial model and parameter selection as $M^{0}$ and $\theta^{0}$

loop $n=0,1, \ldots$ until algorithm convergence

\{ loop $l=0,1, \ldots$ until algorithm convergence or $l=l_{\max }$

$$
\begin{aligned}
& \left\{\text { make } \theta^{n, l+1}=\underset{\theta}{\arg \max } Q\left(\theta: M^{n}, \theta^{n, l}, S\right) ;\right\} \\
& \theta^{n+1}=\theta^{n, l+1} ; \\
& \text { Give the lowest score model between } \\
& \operatorname{Scor}\left(M: \theta^{n}, S\right) \text { and } \operatorname{Scor}\left(M: \theta^{n+1}, S\right) \text { to } M^{n+1} ;
\end{aligned}
$$

Give the parameter of $M^{n+1}$ to $\theta^{n+1, o}$ \}

\section{CONCLUSION}

This chapter discusses the calculation of IDs model selection and maximum utility. To begin with, introducing utility theory and IDs model representation and providing a structural decomposition analysis towards IDs. It resolves IDs into probability network structure and utility structure to reduce the calculated amount of model selection. In addition, providing a PS-MDL which brings in fusion structure prior knowledge to reduce the dependency of troditional MDL towards data. Based on this standard, coming up with PSEM algorithm is used for model selection of probability network structure. Improving computational efficiency of the model selection through calculating parameter learning and structural scoring separately. Meanwhile, for the highdimension of IDs joint utility space, by representing utility function to the sum of each part function to reduce dimension of utility space, form BP neural network used for learning part utility to realize learing of IDs utility structure, and by introducing weight threshold to avoid over-fitting problem. At last, discussing decision-making process based on IDs model for oil Wildcatter model.

\section{REFERENCES}

[1] Madsen, A.L. and D.Nilsson. Solving influence diagrams using HUGIN, Shafer-Shenoy and Lazy propagation[J]. Uncertainty in Artificial Intelligence, 2001,17:337-345

[2] B.Banerjee,A.Bisjee,A.Biswas. Using Bayesian Netoworks to Model Agent Relationships. Applied Artif[J]. Intelligence Journal. 2000,14(9):867-880

[3] S.Noh and P.J.Gmytrasiewicz. Agent modeling in antiarir defense[J]. In Proceedings of the Sixth International Conference on User Modeling. 1997,6: 389-400

[4] Kai Virtanen, Tuomas Raivio. An Influence Diagram Approach to One-on-One Air Combat[J]. 10th on Dynamic Games and Applications (ISDG2002), Russia. 2002, 7: 12-15

[5] Mauricio Marengoni. Decision Making and Uncertainty Management in a 3D Reconstruction System[J]. IEEE Transactions on Pattern Analysis and Machine Intelligence. 2003,25(7) :852-858

[6] Jensen, Finn Verner, Gatti, Elena. Information enhancement-A tool for approximate representation of optimal strategies from influence diagrams[J]. INTERNATIONAL JOURNAL OF APPROXIMATE REASONING.2012, 53(9):1388-1396.

[7] Sedki, Karima, Delcroix. A MODEL BASED ON INFLUENCE DIAGRAMS FOR MULTI-CRITERIA DECISION-MAKING[J]. INTERNATIONAL JOURNAL ON ARTIFICIAL INTELLIGENCE TOOLS.2012, 21(4):18-25.

[8] Fernandez del Rio, Korutcheva, Elka,et al. Interdependent binary choices under social influence: Phase diagram for homogeneous unbiased populations[J]. COMPLEXITY .2012, 16(7):31-41.

[9] Ma ChunLan, Zang TaoCheng, Wang XiaoDan. Hydrostatic pressure influence on magnetic phase diagram and structural parameters of SrTcO3 from first-principles calculations[J]. SCIENCE CHINAPHYSICS MECHANICS \& ASTRONOMY. 2012, 55(7):1253-1257.

[10] Wei Wang, Duo Zhang, Fangyun Lu. The influence of load pulse shape on pressure-impulse diagrams of one-way RC slabs[J]. Structural Engineering and Mechanics. 2012, 42(3):363-81.

[11] Maua, Denis Deratani, Zaffalon, et al. Solving Limited Memory Influence Diagrams[J]. JOURNAL OF ARTIFICIAL INTELLIGENCE RESEARCH. 2012, 44:97-140.

[12] Rotaru, A., Graur, A., Rotaru. Influence of intermolecular interactions and size effect on LITH-FORC diagram in 1D spin crossover compounds[J]. JOURNAL OF OPTOELECTRONICS AND ADVANCED MATERIALS. 2012, 4(5):529-536. 